\title{
A Eubacterial Origin for the Human tRNA Nucleotidyltransferase?
}

\author{
Andreas S. Reichert ${ }^{1,2, \star}$, David L. Thurlow ${ }^{3}$ and \\ Mario Mörl' \\ ${ }^{1}$ Max-Planck-Institute for Evolutionary Anthropology, \\ D-04103 Leipzig, Germany \\ 2 Institute of Physiological Chemistry, University of \\ Munich, D-81377 Munich, Germany \\ ${ }^{3}$ Clark University, Worcester, MA 01610, USA \\ ${ }^{*}$ Corresponding author
}

tRNA CCA-termini are generated and maintained by tRNA nucleotidyltransferases. Together with $\operatorname{poly(A)}$ polymerases and other enzymes they belong to the nucleotidyltransferase superfamily. However, sequence alignments within this family do not allow to distinguish between CCA-adding enzymes and poly(A) polymerases. Furthermore, due to the lack of sequence information about animal CCA-adding enzymes, identification of corresponding animal genes was not possible so far. Therefore, we looked for the human homolog using the baker's yeast tRNA nucleotidyltransferase as a query sequence in a BLAST search. This revealed that the human gene transcript CGI-47 (\#AF151805) deposited in GenBank is likely to encode such an enzyme. To identify the nature of this protein, the cDNA of the transcript was cloned and the recombinant protein biochemically characterized, indicating that CGI-47 encodes a bona fide CCA-adding enzyme and not a poly(A) polymerase. This confirmed animal CCA-adding enzyme allowed us to identify putative homologs from other animals. Calculation of a neighbor-joining tree, using an alignment of several CCAadding enzymes, revealed that the animal enzymes resemble more eubacterial ones than eukaryotic plant and fungal tRNA nucleotidyltransferases, suggesting that the animal nuclear cca genes might have been derived from the endosymbiotic progenitor of mitochondria and are therefore of eubacterial origin.

Key words: CCase/CCA-terminus/tRNA processing.

\section{Introduction}

The generation and maintenance of functional 3'-ends of tRNAs is important for protein translation and cell survival in all organisms. This is accomplished by ATP(CTP):tRNA nucleotidyltransferase, which catalyzes the addition of the trinucleotide CCA to the 3'-OH terminus of tRNAs in a template-independent way. This enzyme is not essential in E. coli, because all of its tRNA genes encode the CCAterminus. However, reduced growth rates and increased amounts of 3'-truncated tRNAs are observed when the corresponding gene cca is inactivated (Deutscher and Hilderman, 1974; Deutscher et al., 1977; Zhu and Deutscher, 1987). In contrast, the enzyme is essential for eukaryotes, archaea, and many other eubacteria, where only very few tRNA genes encode the CCA-terminus (Deutscher, 1990). In budding yeast the essential nature of the cca gene has been shown using a temperaturesensitive tRNA nucleotidyltransferase mutant, which showed growth arrest and an enrichment of 3'-truncated tRNAs upon shifting the cells to the non-permissive temperature (Aebi et al., 1990).

The CCA-adding enzyme belongs to an ancient nucleotidyltransferase superfamily (Holm and Sander, 1995; Martin and Keller, 1996) which is further subdivided into class I and class II enzymes (Yue et al., 1996). According to this scheme, archaeal CCA-adding enzymes, eukaryotic poly $(A)$ polymerases, DNA polymerase $\beta$, kanamycin nucleotidyltransferase, terminal deoxynucleotidyltransferase, and others belong to class I, whereas eubacterial poly(A) polymerases and eubacterial and eukaryotic CCAadding enzymes belong to class II. This reflects, on the one hand, the amazing range of activities comprised by members of this superfamily, but on the other hand it indicates the difficulty associated with predicting the actual function of any newly identified protein sequence of this superfamily. This was exemplified by Raynal and colleagues who showed that the pabS gene, initially thought to code for a poly(A) polymerase in Bacillus subtilis, actually encodes a tRNA nucleotidyltransferase (Raynal et al., 1998).

Despite the important biological function of CCAadding enzymes they have been sequenced and characterized biochemically in only very few species so far, i. e., two eukaryotes (Saccharomyces cerevisiae, Aebi et al., 1990; Lupinus albus, Shanmugam et al., 1996), two eubacteria (Escherichia coli, Cudny et al., 1986; Bacillus subtilis, Raynal et al., 1998) and one archaea (Sulfolobus shibatae, Yue et al., 1996). In animals, only the enzyme from rabbit liver has been studied (Deutscher, 1972a, b); however, its sequence is not known. According to the $\mathrm{NCBI}$ database there are only two more putative eukaryotic tRNA nucleotidyltransferases that have been identified based on amino acid sequence similarities: those from Schizosaccharomyces pombe and Arabidopsis thaliana. Therefore, we started the identification and characterization of the human tRNA nucleotidyltransferase, representing the first homolog from the animal kingdom to be characterized both in terms of sequence and biochemical properties. 


\section{Results}

\section{Identification of a Putative Human tRNA Nucleotidyltransferase}

To search for a homologous tRNA nucleotidyltransferase in humans, the amino acid sequence of tRNA nucleotidyltransferase from S. cerevisiae (Aebi et al., 1990) was used as a query in a BLAST search (Altschul et al., 1990) of human DNA sequences in the NCBI database. The predicted 405 amino acid sequence of the human mRNA CGI-47 (accession no. AF151805) exhibited substantial similarities to the yeast homolog and to other known or putative tRNA nucleotidyltransferases and poly(A) polymerases (data not shown). The CGI-47 protein contains the active site signature (including two highly conserved aspartate residues, $D_{48}$ and $D_{50}$ ) for members of the nucleotidyltransferase family (Holm and Sander, 1995) and can be further classified as a class II nucleotidyltransferase according to Yue et al. (1996). Taken together, the overall conservation of amino acids in the $\mathrm{N}$-terminal part of eubacterial and eukaryotic tRNA nucleotidyltransferases and the CGI-47 protein suggests the latter most likely to be either the human tRNA nucleotidyltransferase or poly(A) polymerase.

\section{Overexpression and Purification of His-Tagged CGI- 47 Protein}

In order to determine whether the CGI-47 protein has the enzymatic activity of a tRNA nucleotidyltransferase, a poly(A) polymerase, or another sort of nucleotidyltransferase, we proceeded with cloning of the gene and overexpression and characterization of its product.
The full-length coding sequence was obtained by RTPCR from total HeLa RNA and cloned into a pET30Ek/Lic vector. Overexpression of the His-tagged CGI-47 protein in E. coli BL21 CodonPlus (DE3)-RP cells was induced with $1 \mathrm{mM}$ IPTG. Samples were taken before induction and one, two and three hours thereafter (Figure 1A, lanes $2,3,4$, and 5, respectively). A prominent band at apparent molecular weight of about 45000 absent before induction is visible already one hour after induction and increases steadily until three hours after induction (Figure 1A). This corresponds approximately to the expected size of $51 \mathrm{kDa}$ for the His-tagged CGI-47 gene product. The crude bacterial extract was loaded onto a His $\bullet$ Bind Quick 900 cartridge (Novagen, Madison, USA), washed with $5 \mathrm{~mm}$ imidazole containing buffer, washed additionally with $60 \mathrm{~mm}$ imidazole buffer and eluted finally with 1 $\mathrm{M}$ imidazole buffer. Aliquots of the washing and elution fractions were separated on a 10\% SDS polyacrylamide gel and proteins were visualized by staining with Coomassie Brilliant Blue (Figure 1B). A majority of the recombinant His-tagged CGI-47 protein with an apparent molecular weight of 45000 already elutes in the $60 \mathrm{~mm}$ imidazole washing step along with some impurities (Figure $1 \mathrm{~B}$, lane 6 ), while the eluate at $1 \mathrm{M}$ imidazole contains only minor impurities (Figure 1B, lanes 8, 9). Therefore, the fraction shown in lane 9 was used for further experiments after desalting.

\section{In Vitro Nucleotide Incorporation Activity of CGI-47 Protein}

To test whether the purified recombinant protein has nucleotide incorporating activity, an in vitro nucleotide incor-
A

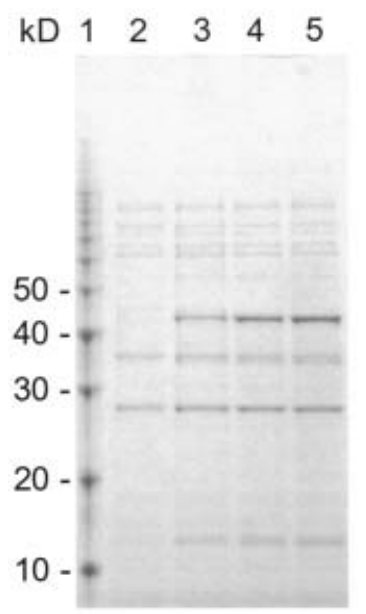

B

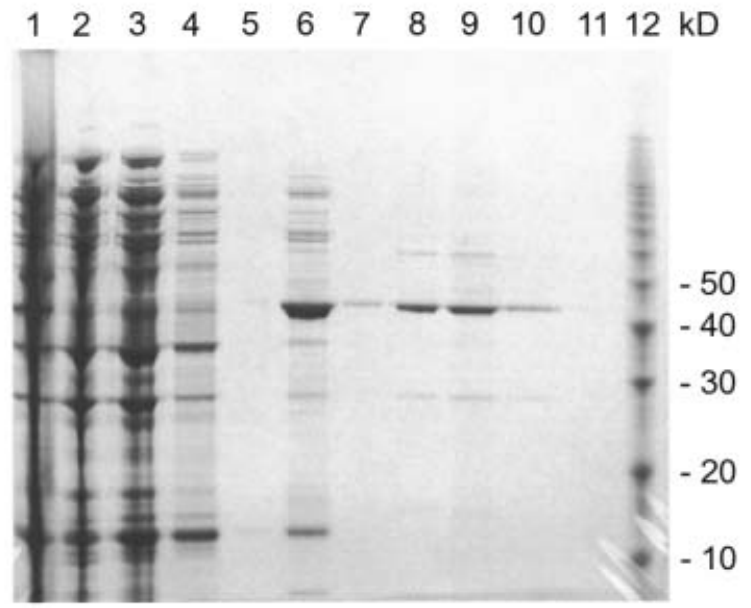

Fig. 1 Overexpression and Affinity Purification of Recombinant Human CGI-47 Protein.

(A) Overexpression of the His-tagged CGI-47 protein in E. coli BL21 CodonPlus (DE3)-RP cells was induced with 1 mM IPTG. Samples were taken before induction and one, two and three hours thereafter (lanes 2, 3, 4, 5, respectively). (B) The overexpressed protein was affinity-purified using a His•Bind Quick 900 cartridge (Novagen). The bacterial extract was loaded onto the cartridge (lane 1), washed with $5 \mathrm{~mm}$ imidazole containing buffer (lanes 2-5), further washed with $60 \mathrm{~mm}$ imidazole containing buffer (lanes 6, 7) and finally eluted with $1 \mathrm{~m}$ imidazole containing buffer (lanes 8 -11). Aliquots of the washing and elution fractions were separated using $10 \%$ SDS PAGE and proteins were visualized by staining with Coomassie Brilliant Blue. Protein from fraction in lane 9 was used for further experiments. 


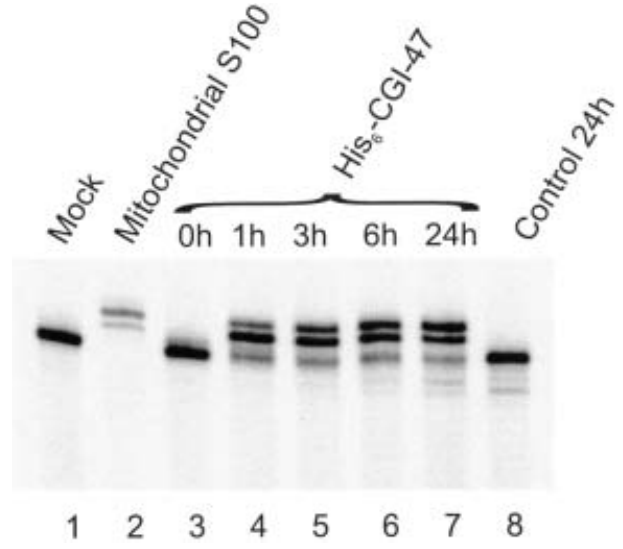

Fig. 2 In Vitro Nucleotide Incorporation Assay.

A $5^{\prime}$ - $\left[{ }^{33} \mathrm{P}\right]$-end-labeled transcript of chimpanzee mitochondrial tRNA ${ }^{\mathrm{Tyr}}$ was incubated in the presence of the four NTPs in the following way: without added protein extract (mock, lane 1); with $3.5-5 \mu \mathrm{g}$ of human mitochondrial protein extract (positive control, lane 2); with $45 \mathrm{ng}$ of overexpressed and His $\bullet$ Bind-purified $\mathrm{His}_{6}-\mathrm{CGl} 47$ protein for $\mathrm{O}$ h (lane 3), $1 \mathrm{~h}$ (lane 4), $3 \mathrm{~h}$ (lane 5), $6 \mathrm{~h}$ (lane 6) and $24 \mathrm{~h}$ (lane 7), and with control protein extract (lane 8). The latter was obtained in parallel to $\mathrm{His}_{6}-\mathrm{CG} / 47$ protein using exactly the same protocol for overexpression and His•Bind-purification but starting from an E. coli BL21-CodonPlus ${ }^{\mathrm{TM} M}$ (DE3)$\mathrm{RP}$ strain carrying a pET30 Lic/EK plasmid with an $32 \mathrm{bp}$ control insert. Incubation was performed for 24 hours at $30^{\circ} \mathrm{C}$ if not indicated otherwise. poration assay was used (Reichert et al., 1998). A 5'-endlabeled chimpanzee mitochondrial tRNA ${ }^{\mathrm{Tyr}}$ substrate was incubated in the presence of all four NTPs for up to $24 \mathrm{~h}$ at $30{ }^{\circ} \mathrm{C}$ with the recombinant $\mathrm{CGI}-47$ protein. The products were analyzed by denaturing PAGE and autoradiography. In the presence of recombinant CGI-47 protein, incorporation of three nucleotides was observed within one hour (Figure 2, lane 4). The intensity of shifted tRNA molecules increases up to six hours of incubation but remains almost unaltered after that (Figure 2, lanes 5-7). As a positive control (Figure 2, lane 2), a human mitochondrial protein extract was used which was shown to incorporate three nucleotides corresponding to the expected CCA addition (Reichert et al., 1998; Reichert and Mörl, 2000). In order to prove that the observed nucleotide incorporating activity originates from the recombinant CGI-47 protein and not from endogenous $E$. coli impurities, we obtained in parallel a control protein extract from the same $E$. coli strain carrying a pET30Lic/Ek control plasmid lacking the CGI-47 coding sequence. Comparable amounts of the extract did not show any nucleotide incorporation even after 24 hours (Figure 2, lane 8).

\section{Enzyme Kinetics of the CGI-47 Protein}

The kinetics of ATP and CTP incorporation reactions of the CGI-47 protein were investigated using the purified enzyme preparation with a specific activity of 65000 units/mg protein (1 unit equals the incorporation of $1 \mathrm{nmol}$ AMP in $1 \mathrm{~h}$ at $37^{\circ} \mathrm{C}$ ). The addition of radioactive

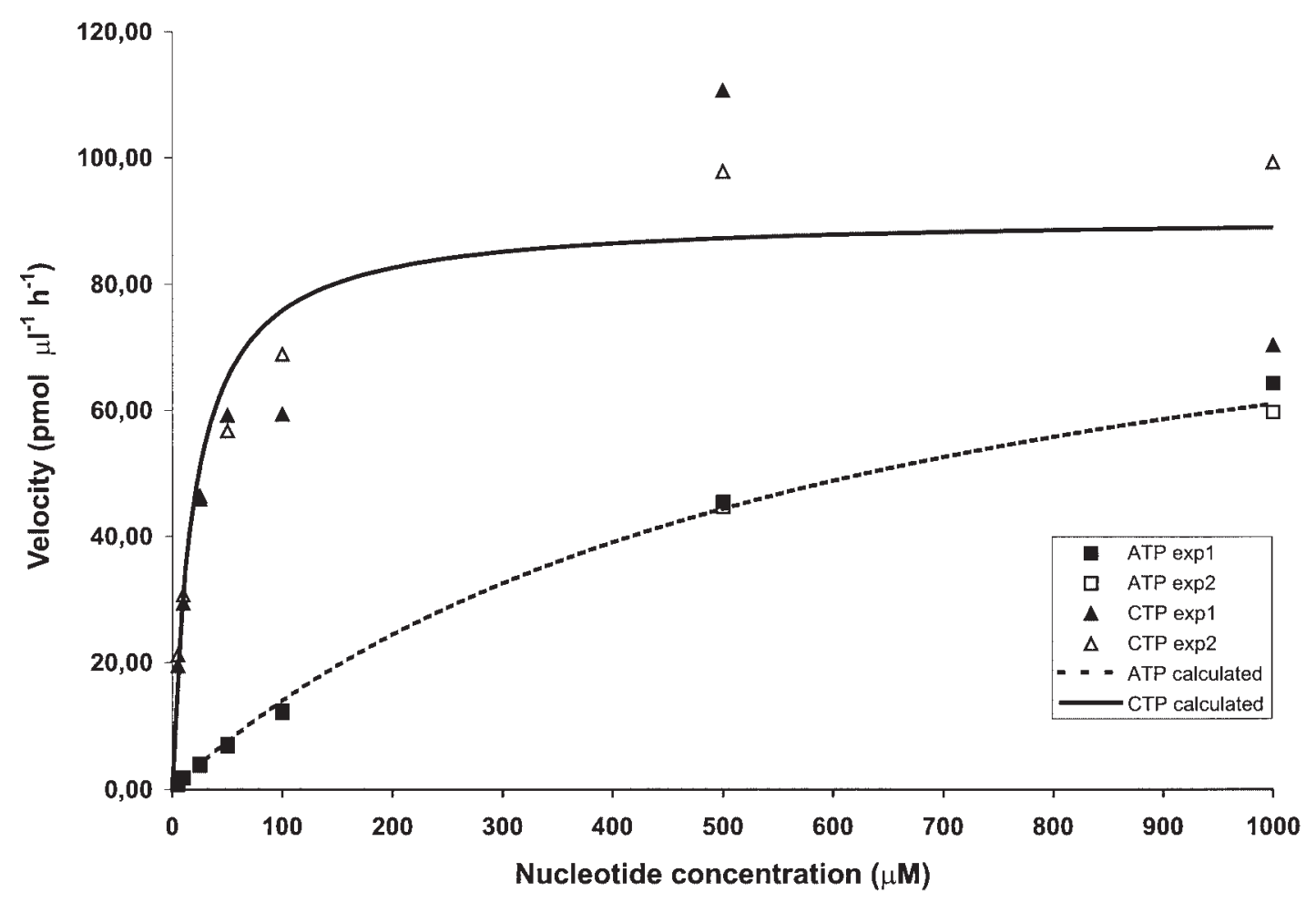

Fig. 3 Enzyme Kinetics of ATP or CTP Incorporation by Human CGI-47 Protein.

The initial velocity of ATP or CTP incorporation by $22.5 \mathrm{ng}$ protein at $37^{\circ} \mathrm{C}$ is given for varying nucleotide concentrations. Two independent experiments for each nucleotide are shown. Furthermore, calculated curves using the average $K_{\mathrm{m}}$ and $V_{\max }$ values obtained for each nucleotide are added. 
AMP or CMP onto bulk $E$. coli tRNA partially missing the CCA-terminus was monitored at varying nucleotide concentrations. Initial velocities follow Michaelis-Menten kinetics for AMP and CMP incorporation (Figure 3), and based on two independent experiments give average $K_{m}$ values of $605 \mu \mathrm{M}$ and $20 \mu \mathrm{M}$, respectively. The $k_{\text {cat }}$ was calculated to be $0.8 \mathrm{~s}^{-1}$. These values are comparable to those known from other tRNA nucleotidyltransferases (Deutscher, 1982). Therefore, we conclude that the CGI-
47 protein indeed represents a human tRNA nucleotidyltransferase and not a poly $(A)$ polymerase.

\section{Identification of Homologous tRNA Nucleotidyl- transferases in C. elegans, D. melanogaster and $M$. musculus}

Using the human tRNA nucleotidyltransferase as a query sequence in a BLASTP search, we obtained candidates

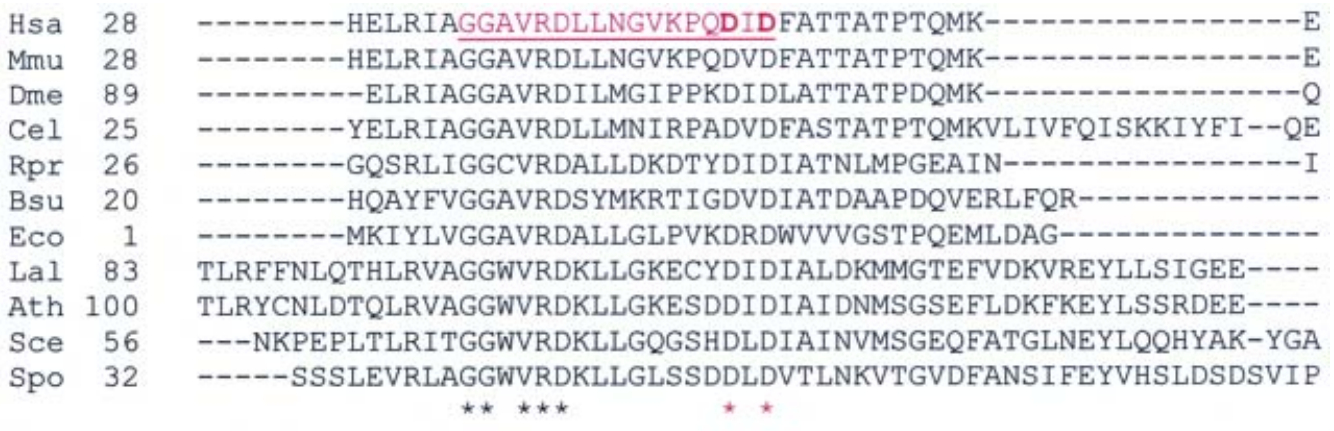

Hsa 62

Mmu 62

Dme 121

Cel 63

Rpr 60

Bsu 58

Eco 39

Lal 132

Ath 149

Sce 102

Spo 76

Hsa 133

Mmu 133

Dme 192

Cel 150

Rpr 130

Bsu 126

Eco 69

Lal 225

Ath 242

Sce 196

Spo 169

Hsa 133

Mmu 133

Dme 192

Cel 150

Rpr 130

Bsu 126

Eco 69

Lal 225

Ath 242

Sce 196

Spo 169
MFQSAGIRMIN-NRGEKHGT ITARLHE-ENFEITTLRI-DVTTDGRHAEVEFTTDWQKDA MFQSAGIRMIN-NKGEKHGT ITARLHE-ENFEVTTLRI-DVTTDGRHAEVEFTTDWQKDA MFEKEEVRMINAN-GEKHGTITPRINDKENFEVTTLRI-DIRTDGRHAEVMYTTDWQLDA MFEEDKIRMLH-KRGEEHGT ITCRIDDCENFEITTLRV-DIVCDGRRAQVEYTTDWQLDA LS-SANIKI I P--TGLKFGTITAILNN-EKFEITTLRK-DIECNGRHAKVIFSKDFAEDA $-----T---V D V G K E H G T I$ IVLWEDETYEVTTFRTESDYVDFRRPSEVQFISSLEEDL $-----Y Q Q V G R D F P V F L H P Q T H E E Y A L A R T E R K S G S G Y T G F T C Y A A P D V T L E D D L$ -AQGVCVIESNPDQSKHLETARMRLFDMWIDFVNLRSE-EYTDNSRI PSMQRFGTPEEDA -VQGDTVIERNPDQSKHLETAKLRIYDQWIDFVNLRSE-EYTENSRIPTM-KFGTAKDDA KPHNIHKIDKNPEKSKHLETATTKLFGVEVDFVNLRSE-KYTELSRI PKV-CFGTPEEDA YKDALGKLTVNPDQSKHLETATLSLFDLDIDFVGLRAE-SYDDKSRIPSV-TPGTVETDA

ERRDLTINSMFLG--FDGTLFDYFNGYEDLKNKKVRFVGHAKQRIQEDYLRILRYFRFYG ERRDLTINSMFLG--FDGTLFDYFNGYADLKNKKVRFVGHAKQRIQEDYLRILRYFRFYG NRRDLTINSMFLG--FDGTVYDYFYGYDDLQERRVVFVGEADIRI KEDFLRILRYFRFYG NRRDLTINSLFLD--LHGNVVDYFGGIRDIETRRIAFVGDARQRIQEDYLRILRYFRFFG ARRDFTINALSYCP-FKNEIYDYFDGFKDLQRAKVVFIGKAFNRI KEDYLRILRFFRFSS KRRDLTINAMAMT--ADGKVLDYFGGKKDIDQKVIRTVGKPEDRFQEDALRMLRAVRFMS KRRDLTINALAQDD--NGEI IDPYNGLGDLQNRLLRHVSPAFG---EDPLRVLRVARFAA YRRDLTINSLFYNINTDSVEDFTKRGISDLKSGKIVTPLPPKATFLDDPLRVVRAIRFGA FRRDLTINSLFYNINSGAVEDLTERGIDDLKSGKIVTPLPAKATFLDDPLRVLRAVRFGA LRRDATLNALFYNI HKGEVEDFTKRGLQDLKDGVLRTPLPAKQTFLDDPLRVLRLIRFAS LRRDFTVNTLFFNIRTEKIEDITKRGYKDLQTKVLVTPISPLQSFLEDPLRILRGIRFAS $* * * * . *$.

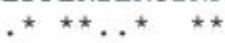

RIVD-KPGDHDPETLEAIAENAK-GLAGISGERIWVELKKILVGNHVNHLIHLI RIVD-RPGDHDHETLEAIAENAK-GLAGISGERIWVELKKILTGDHVNHLIHLI RIAS-EENNHDKATLAAI KENAK-GLARISGERIWSELQKIVPGNFGAALFLEM RISDCTEHEHD--TIQAIVEN-KDGMAGISAERIWTELKKIVVGRMADEVLKAM YYAN----QLD-YESFKACDTLKYGLKTLSRERIKSEIDKI IVSKRATQILEAM QLGFTLSPETEEAIAK---EK--SLLSHVSVERKT IEFEKLLQGRASRQALQTL RYAHLGFRIADETLALMREMTHAGELEHLTPERVWKETESALTTRNPQVFFQVL RFEFT--LDEDLKQAAACDEVKDALAAKISRERIGTEIDLMISGNQPVKAMTYI REGFT--LDEELKEAASSEEVRVALGEKISRERIGNEIDLMISGNGPVSAVTYL RFNET--IDPEVMAEMGDPQINVAFNSKISRERVGVEMEKILVGPTPLLALQLI RFEFT--IDPSVVSAIQDPKVCKAFEKKVSKERVGEEIEKMLKGANAKLALQLL

Fig. 4 Alignment of N-Terminal Protein Sequences of Known or Putative tRNA Nucleotidyltransferases.

Amino acid coordinates are given for the first amino acid on each line. Abbreviations: Hsa: Homo sapiens; Mmu: Mus musculus; Dme: Drosophila melanogaster; Cel: Caenorhabditis elegans; Rpr: Rickettsia prowazekii; Bsu: Bacillus subtilis; Eco: Escherichia coli; Lal: Lupinus albus; Ath: Arabidopsis thaliana; Sce: Saccharomyces cerevisiae; Spo: Schizosaccharomyces pombe. 
from Caenorhabditis elegans (CAB02125), Drosophila melanogaster (AAF51964) and Mus musculus (AK011764) showing 42\% (180/425 amino acids), 49\% (201/404 amino acids), and 94\% (247/261 amino acids) aminoacid identity, respectively. In addition, a second entry of the human CGI-47 sequence (XM_002866) was present in the database showing an N-terminal extension of 29 amino acids, and being otherwise $100 \%$ identical to the CGI-47 protein. Interestingly, this extension is predicted to serve as a cleavable mitochondrial targeting sequence by the program MITOPROT (Claros and Vincens, 1996). The Drosophila homolog also appears to contain an N-terminal mitochondrial targeting sequence, while those from mouse or C. elegans do not. The mouse homolog was derived from a cDNA clone and no function was assigned to it in the NCBI database. The fruit fly and the C. elegans homologs are predicted proteins from the respective complete genome sequences and have been assigned to the poly(A) polymerase family. Nevertheless, it remains unclear whether these proteins are poly $(A)$ polymerases or tRNA nucleotidyltransferases or another member of the nucleotidyltransferase superfamily. At least for the mouse sequence the close similarity to the human tRNA nucleotidyltransferase is striking, as judged by the sequence alignment including protein sequences from Saccharomyces cerevisiae, Schizosaccharomyces pombe, Arabidopsis thaliana, Lupinus albus, Rickettsia prowazekii, Bacillus subtilis and Escherichia coli (Figure 4).

All homologs have the active site signature (underlined in Figure 4) of the nucleotidyltransferase family identified by Holm and Sander (1995) at positions 34-51 (Hsa), which classifies them as class II nucleotidyltransferases (Yue et al., 1996).

In summary, based on the aforementioned biochemical characterization of the human CCA-adding enzyme and the close similarity to this enzyme, we suggest that the mouse, the fruit fly and the $C$. elegans homologs most likely represent tRNA nucleotidyltransferases in these organisms.

\section{Discussion}

\section{CGI-47 Is a CCA-Adding Enzyme, Not a Poly(A) Polymerase}

Following an analysis of sequence alignments of known or suspected tRNA nucleotidyltransferases and a BLASTP search using the yeast enzyme as a query, we hypothesized that the CGI-47 protein is either the human $\operatorname{poly}(A)$ polymerase or the CCA-adding enzyme. This hypothesis was tested by cloning and overexpression of the corresponding gene in E. coli, and by purification and characterization of the gene product. The overexpression system yielded a protein with an additional N-terminal cleavable 43 amino acid His•Tag and S•Tag for detection and purification. Although the expected size of the fusion protein is $51 \mathrm{kDa}$, a major protein product with an apparent molecular weight of about 45000 was observed after induction (Figure 1A). The nucleotide sequence of the cloned insert was confirmed, so the slightly increased electrophoretic mobility in the SDS-PAGE seems to represent a migration artifact. Nevertheless, the enzyme is highly purified as judged by the nearly complete absence of other bands in the gel (Figure 2B, lane 9). An in vitro nucleotide incorporation assay (Figure 2) revealed that the protein is able to add three nucleotides into an in vitro transcribed tRNA substrate missing the CCA-terminus, indicating that the CGI-47 protein is not a poly $(A)$ polymerase but a bona fide tRNA nucleotidyltransferase. Although only two shifted bands are visible, it is evident that these represent tRNA-CC and tRNA-CCA for the following reasons. First, earlier experiments showed that a human mitochondrial $\mathrm{S} 100$ extract, which exhibits the same pattern of reaction product bands as the CGI-47 protein (Figure 2, lane 2), adds three nucleotides (CCA) to this tRNA substrate (Reichert et al., 1998; Reichert and Mörl, 2000). Secondly, using less enzyme an additional band, presumably representing tRNA-C, between the tRNA substrate and the proposed tRNA-CC band was observed (data not shown). Furthermore, one can rule out the possibility that the observed activity results from contaminating endogenous $E$. coli CCA adding enzyme, because a control extract prepared in the same way but missing the $\mathrm{CGl}-47$ gene insert in the cloning vector gave no nucleotide incorporation even after 24 hours of incubation (Figure 2, lane 8).

Another line of biochemical evidence that CGI-47 is a tRNA nucleotidyltransferase comes from the kinetic characterization of the recombinant protein, showing $K_{m}$ values for ATP of $605 \mu \mathrm{M}$ and for CTP of $20 \mu \mathrm{M}$. These values are comparable to those from other organisms in terms of absolute and relative magnitudes (i. e., that for ATP being substantially higher than that for CTP). For example, the enzyme from Sulfolobus shibatae has an apparent $K_{\mathrm{m}}$ for ATP of higher than $200 \mu \mathrm{M}$ and for CTP of 30 uM (Yue et al., 1996).

In summary, the CGI-47 protein represents the first example of an animal CCA-adding enzyme whose sequence is known and which has been characterized biochemically. Since it is not possible to identify the nature and enzymatic properties of a member of the nucleotidyltransferase family solely by sequence comparison, the enzymatic assays provide the only possibility to distinguish between a true tRNA nucleotidyltransferase and a very similar (in terms of sequence) poly(A) polymerase. Therefore, on the basis of our results, we suggest renaming the human CGI-47 protein as a tRNA nucleotidyltransferase.

In order to identify additional enzymes of this class, the human protein sequence was used in a BLASTP search. In addition to homologs in mouse, D. melanogaster, and C. elegans a second human sequence (XM_002866) was obtained from the NCBI database. This protein sequence differs from the first CGI-47 sequence (AF151805) by an additional $\mathrm{N}$-terminal mitochondrial targeting sequence of 29 amino acids. Therefore, it is likely that this protein is 
a mitochondrial tRNA nucleotidyltransferase. If this was the case, it would be possible that the cytoplasmic and the mitochondrial tRNA nucleotidyltransferase originate from the same gene CGI-47 on chromosome 3pter$3 p 25.1$, as was shown to be the case in yeast where one gene encodes for two versions of the enzyme by alternative initiation of translation (Chen et al., 1992; Wolfe et al., 1996). Whether this is also true for humans remains to be determined. One could also imagine that alternative splicing or RNA editing results in different mRNAs coding for these gene products. This idea is supported by the observed sequence differences of the cDNA clones (ESTs) which result from two human full-length mRNA sequences (AF151805 and XM_002866) using different start codons. However, we cannot say at this point whether the sequence differences are due to sequencing errors in the original ESTs or are due to real differences in the transcripts caused by alternative splicing or RNA editing. Furthermore, one cannot exclude the possibility that the nuclear gene encoding the cytoplasmic version of this enzyme has not been detected yet.

The alignment of the N-terminal region of known and newly identified homologs of tRNA nucleotidyltransferases (Figure 4) reveals all enzymes to be members of the class II of the nucleotidyltransferase superfamily according to Yue et al. (1996). In this region, two highly conserved aspartate residues ( $\mathrm{Hsa}, \mathrm{D}_{48}, \mathrm{D}_{50}$; bold and underlined in Figure 4) are part of the active site. Homologous aspartate residues in members of class I nucleotidyltransferases are involved in metal ion coordination (e. $g$. rat DNA polymerase $\beta$; Sawaya et al., 1994). They have also been shown by mutagenesis experiments to be required for catalysis in both poly(A) polymerase (Martin and Keller, 1996) and tRNA nucleotidyltransferase (Yue et al., 1998), demonstrating again the impossibility to predict enzymatic activities in the nucleotidyltransferase superfamily on the basis of protein sequences. Also the conserved RRD-motif (Figure 4) common to most class II nucleotidyltransferases (Yue et al., 1996) is present in all sequences. It is thought to contain the third catalytic aspartate $\left(\mathrm{Hsa}, \mathrm{D}_{123}\right)$ and has also homologs in CCA-adding enzymes $\left(D_{106}\right.$, Thurlow et al., 1997$)$ as well as in poly $(A)$ polymerases $\left(D_{167}\right.$, Martin et al., 1999). Another region of particular interest spans positions 164-175 (Hsa). This has been identified as a highly conserved region, even among archaeal tRNA nucleotidyltransferases, which possibly can adopt an amphipathic $\alpha$-helix (Thurlow et al., 1997). It has also been suggested that this region is involved in nucleotide binding in poly $(\mathrm{A})$ polymerases (Martin et al., 1999). Mutagenesis experiments with the yeast CCA-enzyme confirm this notion (D. L. Thurlow, unpublished results).

\section{Evolution of Animal tRNA Nucleotidyltransferases}

Based on the sequence alignment in Figure 4 we calculated an unrooted neighbor-joining tree (Saitou and Nei, 1987) shown in Figure 5. Although the number of se-

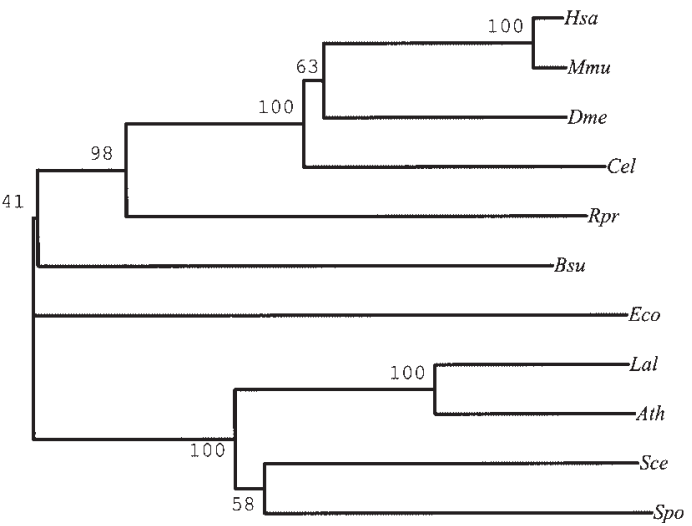

Fig. 5 Phylogenetic Neighbor-Joining Tree of tRNA Nucleotidyltransferases. Abbreviations: see legend to Figure 4. Plant and fungal CCA adding enzymes form a cluster separated from the animal enzymes which are more closely related to that from $R$. prowazekii, E. coli and B. subtilis.

quences used to construct the tree is rather small, several interesting trends emerge. Surprisingly, the animal CCA-adding enzymes are most closely related to that from Rickettsia prowazekii and resemble more the eubacterial enzymes from $E$. coli and $B$. subtilis than the eukaryotic fungal and plant CCA-adding enzymes. The distinction between the animal and plant/fungal groups of enzymes is readily apparent by visual inspection of the sequence alignments (Figure 4).

Mitochondria are believed to originate from an $\alpha$-proteobacterium of the Rickettsia subgroup by endosymbiosis (Gray et al., 1999), suggesting that the animal nuclear cca genes may have been derived from this endosymbiont and encode at least for the mitochondrial CCAadding enzymes in these organisms. Thus, it is feasible that the cca gene was transferred to the nucleus early in animal evolution. However, it is not known whether they have replaced the corresponding eukaryotic gene coding for the nuclear and cytoplasmic tRNA nucleotidyltransferase or whether the latter has not been identified yet (see above). At least for the Drosophila and the human homologs, a mitochondrial targeting sequence has been identified, thereby directing the gene product back to mitochondria. In contrast, the plant and fungal CCA-adding enzymes identified so far form a discrete cluster clearly separated from the animal enzymes and do not seem to have originated from this endosymbiotic event.

Taken together, these results suggest that animal tRNA nucleotidyltransferases (including the human enzyme) are likely to have been derived from enzymes that were taken over by the eukaryotic host of the mitochondrial progenitor.

\section{Materials and Methods}

\section{Cloning and Overexpression}

Using total RNA prepared from HeLa cells (a gift from Wolfgang Enard and Florian Heissig) CDNA was prepared using Oligo(dT) 
priming and Superscript II reverse transcriptase according to the supplier (Gibco/BRL, Rockville, USA). The full-length coding sequence (1218 bp) was PCR amplified (2 min at $94^{\circ} \mathrm{C}$; 30 cycles $1 \mathrm{~min}$ at $94^{\circ} \mathrm{C} / 1 \mathrm{~min}$ at $50^{\circ} \mathrm{C} / 3 \mathrm{~min}$ at $72{ }^{\circ} \mathrm{C} ; 5 \mathrm{~min}$ at $72^{\circ} \mathrm{C}$ ) with the following primers: hscca1 (5'-GAC GAC GAC AAG ATG AAG TTG CAG TCT CCC G-3') and hscca2 (5'-GAG GAG AAG CCC GGT TAG GTC TTC TTT ATG TAA CTC-3'). The PCR product was cloned into pET30 Ek/Lic according to the supplier (Novagen, Madison, USA). This resulted in a construct with additional $43 \mathrm{~N}$-terminal cleavable amino acid (His•Tag and S•Tag). Initial transformation was performed by electroporation into $E$. coli TOP10 One Shot cells according to the supplier (Invitrogen, Carlsbad, USA). Subsequently, the plasmid DNA from a positive clone was transformed into E. coli BL21-CodonPlus (DE3)-RP for overexpression. DNA sequencing of this plasmid DNA on an ABI Prism 377 automated sequencer confirmed the deposited sequence with the exception of two silent mutations (TTT $\rightarrow$ TTC, coding for $\mathrm{Phe}_{111}$ and GCG $\rightarrow$ GCA, coding for $A \mathrm{Al}_{287}$ ). An overnight culture of $E$. coli BL21-CodonPlus ${ }^{\mathrm{TM}}$ (DE3)-RP/pET30 Lic/EK-hscca\#7 cells was used to inoculate $250 \mathrm{ml}$ LB supplemented with kanamycin $(30 \mu \mathrm{g} / \mathrm{ml})$ and chloramphenicol (34 $\mu \mathrm{g} / \mathrm{ml})$. The culture was grown at $37^{\circ} \mathrm{C}(120 \mathrm{rpm}$ shaking) to an $\mathrm{OD}_{600 \mathrm{~nm}}$ of 1.0. Expression of the recombinant human tRNA nucleotidyltransferase was then induced by the addition of IPTG to a final concentration of $1 \mathrm{~mm}$. After three hours of induction, the cells were left on ice for five minutes and subsequently harvested by centrifugation at $5000 \mathrm{~g}$ for 10 minutes at $4{ }^{\circ} \mathrm{C}$. The bacterial pellet was stored at $-80^{\circ} \mathrm{C}$ until use.

Affinity purification of the recombinant His-tagged protein was done according to the supplier using the His-Bind Kit (Novagen): the cells were thawed on ice and resuspended in $4 \mathrm{ml}$ binding buffer (5 mm imidazol, $0.5 \mathrm{~m} \mathrm{NaCl}, 20 \mathrm{~mm}$ Tris/Cl pH 7.9) and sonicated with a MS-73mm tip on a Sonopuls HD 2070 sonicator (Bandelin, Berlin, Germany) 10 times each for $15 \mathrm{~s}$ on ice. The volume was adjusted to $15 \mathrm{ml}$ and centrifuged (20 min, $18000 \mathrm{rpm}$ in a SS34 rotor, $\left.39000 \mathrm{~g}, 4^{\circ} \mathrm{C}\right)$. An aliquot $(2 \mathrm{ml})$ of the supernatant was loaded onto an equilibrated His $\bullet$ Bind Quick 900 cartridge. After washing with $20 \mathrm{ml}$ binding buffer and $10 \mathrm{ml}$ wash buffer $(60 \mathrm{mM}$ imidazole, $0.5 \mathrm{M} \mathrm{NaCl}, 20 \mathrm{~mm}$ Tris/Cl pH 7.9) the His-tagged protein was eluted with elution buffer ( $1 \mathrm{~m}$ imidazole, $0.5 \mathrm{M} \mathrm{NaCl}, 20 \mathrm{~mm}$ Tris/Cl pH 7.9) in four fractions, each containing $1 \mathrm{ml}$. Before assaying the fractions for activity they were desalted with G25 microspin columns (Amersham Pharmacia Biotech, Freiburg, Germany) equilibrated in $20 \mathrm{~mm} \mathrm{HEPES/KOH} \mathrm{(pH7,9),} 1$ mm DTT.

\section{Preparation of Substrate tRNA}

The template for in vitro transcription was prepared by PCR amplification $\left(2 \mathrm{~min}\right.$ at $94{ }^{\circ} \mathrm{C}$; 30 cycles $1 \mathrm{~min}$ at $94{ }^{\circ} \mathrm{C} / 1 \mathrm{~min}$ at $50{ }^{\circ} \mathrm{C} / 30 \mathrm{~s}$ at $72{ }^{\circ} \mathrm{C}$ ) of the corresponding mitochondrial gene using the following primers:

T7(chimpTyr): 5'-CTAATACGACTCACTATAGGTAAAATGGCTGAG-3'.

Downstream(chimpTyr): 5'-TGGTAAAAAGAGGCTTAA-3'.

Transcription with T7 RNA polymerase (New England Biolabs, Schwalbach, Germany), dephosphorylation using calf intestinal alkaline phosphatase (New England Biolabs) and 5'-end-labeling using polynucleotide kinase (New England Biolabs) and $[\gamma-$ ${ }^{33}$ P]-ATP (Amersham Pharmacia Biotech, Freiburg, Germany) were carried out according to the supplier. Radioactively labeled transcripts or reaction products were separated and purified by denaturing polyacrylamide gel electrophoresis (Peattie, 1979).

\section{In Vitro Nucleotide Incorporation Assay}

5'-[ $\left.{ }^{33} \mathrm{P}\right]$-labeled tRNA substrate $(1-10 \mathrm{pmol})$ was incubated with $45 \mathrm{ng}$ of highly purified human CGI-47 protein in the presence of
1 mm NTPs, 30 mm HEPES-KOH ( $\mathrm{pH} 7.6$ ), 6 mm $\mathrm{MgCl}_{2}, 30 \mathrm{~mm}$ $\mathrm{KCl}, 2 \mathrm{~mm}$ DTT and $30 \mathrm{U}$ RNase inhibitor (Roche Molecular Biochemicals, Mannheim, Germany) in a total volume of $20 \mu \mathrm{l}$ for up to 24 hours at $30^{\circ} \mathrm{C}$. After ethanol precipitation, the products were separated by electrophoresis on a $10 \%$ polyacrylamide gel containing $8 \mathrm{~m}$ urea and visualized by autoradiography.

\section{Enzyme Assays}

The standard assay $(15 \mu \mathrm{l})$ contained $50 \mathrm{~mm}$ glycine $(\mathrm{pH} 9.4)$, $20 \mathrm{~mm} \mathrm{MgCl} 2,1 \mathrm{~mm}$ dithiothreitol (DTT), $30 \mu \mathrm{m} \mathrm{tRNA}$, and ATP or CTP $(5-1000 \mu \mathrm{M})$. The tRNA substrate was total tRNA of $E$. coli (Sigma, St. Louis, USA), from which the CCA sequence had been partially removed (Deutscher, 1972a) by treatment with snake venom phosphodiesterase (Worthington Chemical, Freehold, USA). Addition of AMP or CMP onto intact tRNA was monitored by including $0.33 \mu \mathrm{M}\left[\alpha^{-32} \mathrm{P}\right]$-ATP or $\left[\alpha^{-32} \mathrm{P}\right]-\mathrm{CTP}(3000 \mathrm{Ci}$ per mmol; ICN Pharmaceuticals Inc., Costa Mesa, USA). For titration of NTP, $30 \mu \mathrm{M}$ tRNA and NTP at final concentrations ranging from 5-1000 $\mu \mathrm{M}$ were used, and $100 \mu \mathrm{m}$ unlabeled CTP was included for the ATP titration. Following pre-incubation at $37^{\circ} \mathrm{C}$ for $30 \mathrm{~s}$, the reaction was initiated by adding enzyme $(0.032 \mu \mathrm{M})$ and was continued for $5 \mathrm{~min}$ at $37^{\circ} \mathrm{C}$. The reaction was stopped by adding $5 \mu \mathrm{l}$ of formamide containing $0.025 \%$ xylene cyanole and $0.025 \%$ bromophenol blue and transfering the reaction to ice. The mixture was loaded onto a denaturing $10 \%$ polyacrylamide gel (acrylamide:N,N'-methylene bisacrylamide:19:1) in $100 \mathrm{~mm}$ Tris-borate (pH 8.3), $2.5 \mathrm{~mm}$ EDTA, and 8 $\mathrm{M}$ urea. Intact $\left[{ }^{32} \mathrm{P}\right]$-labeled tRNA was visualized by autoradiography, sliced from the gel, and counted in liquid scintillation cocktail using a Packard 1900CA liquid scintillation counter. Specific activities of the labeled NTP were used to convert radioactivity in gel slices to moles of incorporated nucleotide. They were determined by counting an aliquot of the incubation mix polymerized into pieces of polyacrylamide, comparable in size and thickness to slices from the assay gel, and dividing this measured amount of radioactivity by the amount of total NTP in the same volume of incubation mix. All radioactivity measurements were corrected for decay to the manufacturer's assay date of the isotope.

Apparent Michaelis-Menten constants $\left(K_{m}\right)$ and maximum velocities $\left(V_{\max }\right)$ were calculated using a least-squares curve fit to the Michaelis-Menten equation (Kaleidagraph, Ablebeck Software), and statistically, assuming that each measured initial velocity is subject to the same proportion of random error (Cornish-Bowden and Wharton, 1988). Both approaches gave similar estimates, and kinetic constants are reported as the average of calculated values for all titrations using both approaches \pm one standard deviation from the mean. The calculated value of $V_{\max }(\mu \mathrm{m} / \mathrm{s})$ was divided by the enzyme concentration $(0.032 \mu \mathrm{M})$ to obtain an estimate of $k_{\text {cat }}$.

\section{Sequence Alignment and Analysis}

The N-terminal regions of the known or putative CCA-adding enzymes from Bacillus subtilis (Bsu, Z99115), Escherichia coli (Eco, M12788), Arabidopsis thaliana (Ath, AC003979), Lupinus albus (Lal, U15930), Saccharomyces cerevisiae (Sce, M59870), Schizosaccharomyces pombe (Spo, AL049498), Rickettsia prowazekii (Rpr, AJ235270), Homo sapiens (Hsa, (AF151805), Caenorhabditis elegans (Cel, CAB02125), Mus musculus (Mmu, AK011764) and Drosophila melanogaster (Dme, AAF51964) were aligned according to the Smith and Waterman algorithm using a gap penalty of 10 and a gap extension penalty of 0.05 (Smith and Waterman, 1981; Thompson et al., 1994). The sequence from Rickettsia prowazekii is classified as eubacterial poly $(\mathrm{A})$ polymerases (PAP) based on sequence similarities with 
the corresponding protein from Bacillus subtilis. However, the Bacillus protein has been purified and characterized biochemically as a tRNA nucleotidyltransferase (Raynal et al., 1998), and it is therefore likely that the putative PAP, identified as such by similarity to the Bacillus enzyme, is also a CCA-adding enzyme. Accordingly, it was included in the present study. For predicting the presence of a $\mathrm{N}$-terminal mitochondrial targeting sequence the MITOPROT program at www.mips.biochem.mpg.de/proj/ medgen/mitop/ was used (Claros and Vincens, 1996). The neighbor-joining tree was based on the distance matrix from the alignment in Figure 4 with 10000 bootstrapping iterations (Saitou and Nei, 1987).

\section{Acknowledgements}

This work was supported by the Max-Planck-Society and the National Institutes of Health (grant 1R15GM57685-01).

\section{References}

Aebi, M., Kirchner, G., Chen, J.Y., Vijayraghavan, U., Jacobson, A., Martin, N.C., and Abelson, J. (1990). Isolation of a temperature-sensitive mutant with an altered tRNA nucleotidyltransferase and cloning of the gene encoding tRNA nucleotidyltransferase in the yeast Saccharomyces cerevisiae. J. Biol. Chem. 265, 16216-16220.

Altschul, S.F., Gish, W., Miller, W., Myers, E.W., and Lipman, D.J. (1990). Basic local alignment search tool. J. Mol. Biol. 215 , 403-410.

Chen, J.Y., Joyce, P.B., Wolfe, C.L., Steffen, M.C., and Martin, N.C. (1992). Cytoplasmic and mitochondrial tRNA nucleotidyltransferase activities are derived from the same gene in the yeast Saccharomyces cerevisiae. J. Biol. Chem. 267, $14879-14883$

Claros, M.G., and Vincens, P. (1996). Computational method to predict mitochondrially imported proteins and their targeting sequences. Eur. J. Biochem. 241, 779-786.

Cornish-Bowden, A., and Wharton, C.W. (1988). Enzyme Kinetics (Oxford, UK: IRL Press).

Cudny, H., Lupski, J.R., Godson, G.N., and Deutscher, M.P. (1986). Cloning, sequencing, and species relatedness of the Escherichia coli cca gene encoding the enzyme tRNA nucleotidyltransferase. J. Biol. Chem. 261, 6444-6449.

Deutscher, M.P. (1972a). Reactions at the 3' terminus of transfer ribonucleic acid. 3. Catalytic properties of two purified rabbit liver transfer ribonucleic acid nucleotidyl transferases. J. Biol. Chem. 247, 459-468.

Deutscher, M.P. (1972b). Reactions at the 3' terminus of transfer ribonucleic acid. II. Purification and physical and chemical properties of rabbit liver transfer ribonucleic acid nucleotidyltransferase. J. Biol. Chem. 247, 450-458.

Deutscher, M.P. (1982). tRNA Nucleotidyltransferase. Enzymes 15, $183-215$.

Deutscher, M. (1990). Ribonucleases, tRNA nucleotidyltransferase, and the 3'processing of tRNA. Prog. Nucl. Acid Res. Mol. Biol. 39, 209-240.

Deutscher, M.P., and Hilderman, R.H. (1974). Isolation and partial characterization of Escherichia coli mutants with low levels of transfer ribonucleic acid nucleotidyltransferase. J. Bacteriol. 118, 621-627.

Deutscher, M.P., Lin, J.J., and Evans, J.A. (1977). Transfer RNA metabolism in Escherichia coli cells deficient in tRNA nucleotidyltransferase. J. Mol. Biol. 117, 1081-1094.
Gray, M.W., Burger, G., and Lang, B.F. (1999). Mitochondrial evolution. Science 283, 1476-1481.

Holm, L., and Sander, C. (1995). DNA polymerase $\beta$ belongs to an ancient nucleotidyltransferase superfamily. Trends Biochem. Sci. 20, 345-347.

Martin, G., and Keller, W. (1996). Mutational analysis of mammalian poly $(A)$ polymerase identifies a region for primer binding and catalytic domain, homologous to the family $X$ polymerases, and to other nucleotidyltransferases. EMBO J. 15, 2593-2603.

Martin, G., Jeno, P., and Keller, W. (1999). Mapping of ATP binding regions in poly $(A)$ polymerases by photoaffinity labeling and by mutational analysis identifies a domain conserved in many nucleotidyltransferases. Protein Sci. 8, 2380-2391.

Peattie, D.A. (1979) Direct chemical method for sequencing RNA. Proc. Natl. Acad. Sci. USA 76, 1760-1764.

Raynal, L.C., Krisch, H.M., and Carpousis, A.J. (1998). The Bacillus subtilis nucleotidyltransferase is a tRNA CCA-adding enzyme. J. Bacteriol. 180, 6276-6282.

Reichert, A.S., and Mörl, M. (2000). Repair of tRNAs in metazoan mitochondria. Nucleic Acids Res. 28, 2043-2048.

Reichert, A., Rothbauer, U., and Mörl, M. (1998). Processing and editing of overlapping tRNAs in human mitochondria. J. Biol. Chem. 273, 31977-31984.

Saitou, N., and Nei, M. (1987). The neighbor-joining method: a new method for reconstructing phylogenetic trees. Mol. Biol. Evol. 4, 406-425.

Sawaya, M.R., Pelletier, H., Kumar, A., Wilson, S.H., and Kraut, J. (1994). Crystal structure of rat DNA polymerase $\beta$ : evidence for a common polymerase mechanism. Science 264, $1930-1935$.

Shanmugam, K., Hanic-Joyce, P.J., and Joyce, P.B. (1996). Purification and characterization of a tRNA nucleotidyltransferase from Lupinus albus and functional complementation of a yeast mutation by corresponding cDNA. Plant Mol. Biol. 30, $281-295$

Smith, T.F., and Waterman, M.S. (1981). Identification of common molecular subsequences. J. Mol. Biol. 147, 195-197.

Thompson, J.D., Higgins, D.G., and Gibson, T.J. (1994). CLUSTAL W: improving the sensitivity of progressive multiple sequence alignment through sequence weighting, positionspecific gap penalties and weight matrix choice. Nucleic Acids Res. 22, 4673-4680.

Thurlow, D.L., Pulido, G.M., and Millar, K.J. (1997). Unidentified open reading frames in the genome of Methanococcus jannaschii are similar in sequence to an archaebacterial gene for tRNA nucleotidyltransferase. J. Mol. Evol. 44, 686-689.

Wolfe, C.L., Hopper, A.K., and Martin, N.C. (1996). Mechanisms leading to and the consequences of altering the normal distribution of ATP(CTP):tRNA nucleotidyltransferase in yeast. J. Biol. Chem. 271, 4679-4686.

Yue, D., Maizels, N., and Weiner, A.M. (1996). CCA-adding enzymes and poly $(A)$ polymerases are all members of the same nucleotidyltransferase superfamily: characterization of the CCA-adding enzyme from the archaeal hyperthermophile Sulfolobus shibatae. RNA 2, 895-908.

Yue, D., Weiner, A.M., and Maizels, N. (1998). The CCA-adding enzyme has a single active site. J. Biol. Chem. 273, 29693-29700.

Zhu, L., and Deutscher, M.P. (1987). tRNA nucleotidyltransferase is not essential for Escherichia coli viability. EMBO J. 6, $2473-2477$.

Received June 25, 2001; accepted August 8, 2001 